\author{
Yu. V. Korotkyi ${ }^{1}$, D. M. Dudikova ${ }^{2}$, N. O. Vrynchanu'2, O. A. Smertenko ${ }^{1}$ \\ ${ }^{1}$ Institute of Organic Chemistry of NAS of Ukraine \\ ${ }^{2}$ SI "Institute of Pharmacology and Toxicology of NAMS of Ukraine"
}

\title{
SYNTHESIS AND BIOLOGICAL ACTIVITY OF NOVEL ADAMANTANE-BASED DIALKYLAMINOPROPANOL QUATERNARY SALTS
}

Topicality. The emergence and spread of multidrug-resistant pathogens leads to a decrease in efficacy of antibiotic therapy, causes the duration of patient's hospital stay and increases treatment costs. The screening of potential antimicrobial agents among the new classes of chemical compounds is one of the promising methods to overcome the problem of resistance.

Aim. To synthesize and to make screening studies of antimicrobial activity of quaternary salts of adamantane derivatives (3a-3l) with the aim to find of new prospective compound with good activity.

Materials and methods. The synthesis and investigation of physicochemical properties of new adamantane-based dialkylaminopropanol quaternary salts were carried out. The evaluation of antimicrobial action against S. aureus, E. coli and C. albicans strains were performed.

Results and discussion. The results showed that the inhibitory activities of quaternary salts with 1-adamantylethyl radical in their alkoxy group were significantly higher than those of the compounds with 1-adamantyl and 1-adamantyloxyethyl radicals in their alkoxy group.

Conclusions. 3c was the most active compound tested against all strains, with MIC between 1.56 and $3.12 \mu \mathrm{g} / \mathrm{mL}$, and its antimicrobial activity was similar to that of myramistin.

Key words: adamantane derivatives; dialkylaminopropanol; synthesis; antibacterial activity; antifungal action

Ю. В. Короткий, Д. М. Дудікова, Н. О. Вринчану, О. А. Смертенко Синтез та біологічна активність нових четвертинних солей адамантановмісних діалкіламінопропанолів

Актуальність. Поява та розповсюдження стійких штамів збудників інфекційних хвороб призводить до зниження ефективності антибіотикотерапії, спричиняє збільшення терміну госпіталізації пацієнтів та витрат на їх лікування. Одним із шляхів протидії резистентності $€$ пошук активних сполук серед нових хімічних класів та розробка на їх основі ефективних препаратів.

Мета роботи. Синтез та скринінгові дослідження антимікробної дії четвертинних солей адамантановмісних діалкіламінопропанолів (3a-3l) з метою пошуку нових перспективних активних сполук.

Матеріали та методи. Здійснено синтез та вивчені фізико-хімічні властивості четвертинних солей адамантановмісних діалкіламінопропанолів. Було оцінено їх антимікробну дію відносно S. aureus, E. coli та C. albicans.

Результати та їх обговорення. Було встановлено, що інгібуюча активність четвертинних солей з 1-адамантилетиловим радикалом у алкоксигрупі була значно вищою, ніж у сполук з 1-адамантиловим та 1-адамантилоксіетильним радикалами.

Висновки. Найбільш виражену інгібуючу активність по відношенню до всіх штамів показала сполука 3с, яка за антимікробною активністю не поступалась препарату порівняння мірамістину (МІК був в інтервалі 1,56-3,12 мкг/мл).

Ключові слова: похідні адамантану; діалкіламінопропаноли; синтез; антибактеріальна активність; протигрибкова дія

Ю. В. Короткий, Д. М. Дудикова, Н. А. Врынчану, Е. А. Смертенко

Синтез и биологическая активность новых четвертичных солей адамантансодержащих диалкиламинопропанолов

Актуальность. Появление и распространение устойчивых штаммов возбудителей инфекционных заболеваний приводит к снижению эффективности антибиотикотерапии, способствует увеличению срока госпитализации пациентов и расходов на их лечение. Одним из путей борьбы с резистентностью является поиск активных соединений среди новых химических классов и разработка на их основе эффективных препаратов.

Цель работы. Синтез и скрининговые исследования антимикробного действия четвертичных солей адамантансодержащих диалкиламинопропанолов с целью поиска новых перспективных активных соединений.

Материалы и методы. Осуществлен синтез и изучены физико-химические свойства четвертичных солей адамантансодержащих диалкиламинопропанолов. Была проведена оценка их антимикробного действия в отношении S. aureus, E. coli и C. albicans.

Результаты и их обсуждение. Показано, что ингибирующая активность четвертичных солей с 1-адамантилэтильным радикалом в алкоксигруппе была значительно выше, чем у соединений с 1-адамантильным и 1-адамантилоксиэтильным радикалами.

Выводы. Наиболее выраженная активность в отношении всех штаммов была установлена у соединения 3c, которое по показателю антимикробной активности не уступает препарату сравнения мирамистину (МПК был в диапазоне 1,56-3,12 мкг/мл).

Ключевые слова: производные адамантана; диалкиламинопропанолы; синтез; антибактериальная активность; противогрибковое действие 


\section{INTRODUCTION}

Nowadays, the emergence and spread of multidrugresistant (MDR) pathogens (e.g. methicillin-resistant Staphylococcus aureus (MRSA), carbapenem-resistant Enterobacteriaceae (CRE), fluoroquinolone-resistant Escherichia coli or resistant to third-generation cephalosporins Neisseria gonorrhoeae isolates) pose a global threat to public health. According to WHO data about antibiotic-resistance (2014), the lethality in patients with MRSA infection is higher on $64 \%$ than with susceptible form of infection [1]. A wide spread of MDR-pathogens leads to a decrease in efficacy of antibiotic therapy, prolongation of the length of patient's hospital stay and an increase in treatment costs. In addition, patients with MDR-infections often have increased mortality rates.

The screening of potential antimicrobial agents among the new classes of chemical compounds is one of the promising methods to overcome the problem of resistance. In this respect, adamantane-based aminopropanols are attracting attention owing to their broad antibacterial and antifungal activity $[2,3]$. The significant antimicrobial action is attributable to high lipophilicity and crystal structure of the adamantane moiety [4].

The aim is to carry out synthesis and screening studies of antimicrobial activity of quaternary salts of adamantane derivatives (3a-3l) with the aim to find of new prospective compound with good activity.

\section{MATERIALS AND METHODS}

The structure of compounds was confirmed by using set of physical and chemical methods such as elemental analysis, IR- and ${ }^{1} \mathrm{H}$ NMR-spectrometry. The elemental analyses were detected using a Carlo Erba CHNS-OEA 1106 analyzer. The melting point were determined on a Gallenkamp melting point apparatus MFB-595 in open capillary tube. ${ }^{1} \mathrm{H}$ NMR-spectra were acquired on a Varian VXP spectrometer (299.945 MHz), the solvent was DMSO- $d_{6}$ with tetramethylsilane (TMS) as an internal standard. IR spectra were registered by using UR-20 spectrophotometer with liquid films between $\mathrm{KBr}$ plates.
Antimicrobial activity of synthesized compounds (3a-3l) was assayed by serial twofold dilution method [5] against gram-positive (Staphylococcus aureus ATCC 25923) and gram-negative (Escherichia coli ATCC 25922) bacteria and yeasts (Candida albicans NCTC 885/653). Inoculum density was $1-2 \times 10^{5} \mathrm{CFU} / \mathrm{ml}$ culture medium (bacteria) and $1-2 \times 10^{4} \mathrm{CFU} / \mathrm{ml}$ (yeasts).The 96 -well microtiter plates with bacterial cultures were incubated at $35-37{ }^{\circ} \mathrm{C}$ for 18-24 h, with yeasts - at $30-32{ }^{\circ} \mathrm{C}$ for $24-48 \mathrm{~h}$. MuellerHinton broth ( $\mathrm{pH}$ 7.2) (HiMedia) and Saburo dextrose broth ( $\mathrm{pH}$ 5.6) (HiMedia) were used for minimal inhibitory concentration (MIC) determination. Myramistin was used as reference substance. The lowest concentration of compounds, that inhibited microbial growth, was considered as the MIC. All assays were conducted in triplicate with controls of culture growth (as positive) and cultural media (as negative).

\section{RESULTS AND DISCUSSION \\ Experimental Chemical Part}

In our work 1-adamantylglycidyl ether, 1-adamantylethoxy- and 1-(2-adamantoxyethoxy)-glycidyl ethers were used as initial substances. 1-Adamantylglycidyl ether was obtained according to the method reported previously [6]; 1-adamantylethoxy and 1-(2-adamantoxyethoxy) glycidyl ethers were obtained under conditions of the phase-transfer catalysis $(50 \% \mathrm{NaOH}, \mathrm{TEBAC}$, epichlorohydrin) (Scheme) [7].

The synthesis of the target compounds was carried out according to Scheme 1. Quaternary salts of adamantane-based dialkylaminopropanol (3a-1) were obtained starting from relevant epoxides (1) by treatment with excess amount (double amount, $50 \%$ ) of secondary amines in the presence of isopropanol with heating. The excess of reagents (amines and alcohol) was evaporated under reduced pressure. Then these intermediates (2) were converted into quaternary salts (3a-l) by treatment with excess amount (5\%) of alkyl halides $\left(\mathrm{CH}_{3} \mathrm{I}, \mathrm{C}_{2} \mathrm{H}_{5} \mathrm{I}\right.$, $\mathrm{C}_{6} \mathrm{H}_{5} \mathrm{CH}_{2} \mathrm{Cl}$ ) in the presence acetone or acetonitrile with heating at $10 \mathrm{~h}$.

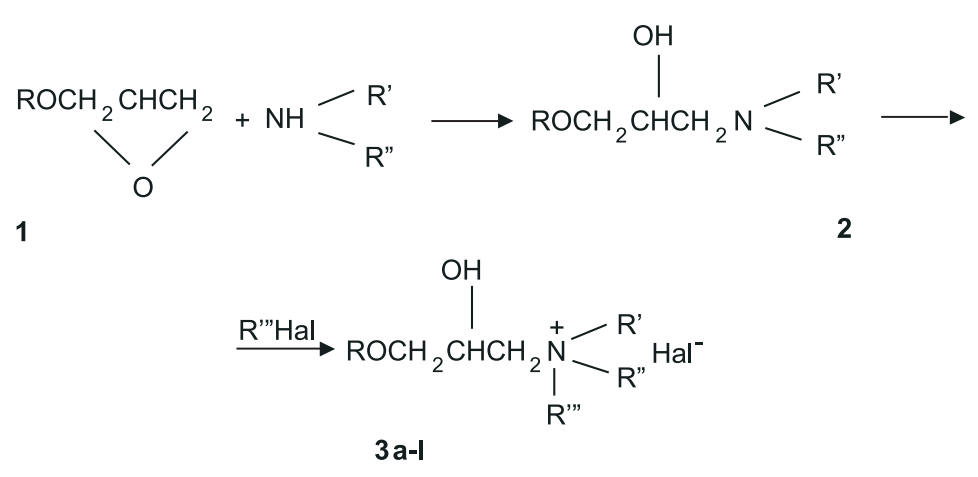

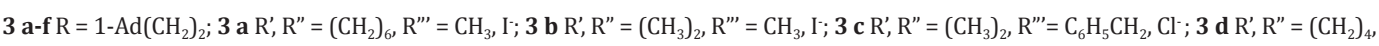
$\mathrm{R}^{\prime \prime \prime}=\mathrm{CH}_{3}, \mathrm{I} ; \mathbf{3}$ e R', R" = $\left(\mathrm{CH}_{2}\right)_{4}, \mathrm{R}^{\prime \prime \prime}=\mathrm{C}_{6} \mathrm{H}_{5} \mathrm{CH}_{2}, \mathrm{Cl} ; \mathbf{3} \mathbf{f} \mathrm{R}^{\prime}, \mathrm{R}^{\prime \prime}=\left(\mathrm{CH}_{2}\right)_{6}, \mathrm{R}^{\prime \prime}=\mathrm{C}_{6} \mathrm{H}_{5} \mathrm{CH}_{2}, \mathrm{Cl} ; \mathbf{3}$ g-j R = 1-adamantyl; 3 g R', R" = $\left(\mathrm{CH}_{2}\right)_{4}, \mathrm{R}^{\prime \prime \prime}=\mathrm{CH}_{3}, \mathrm{I} ; \mathbf{3}$ h R',

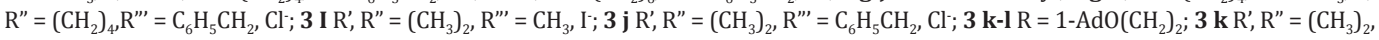
$\mathrm{R}^{\prime \prime \prime}=\mathrm{CH}_{3}, \mathrm{I} ; 31 \mathrm{R}, \mathrm{R}^{\prime \prime}=\left(\mathrm{CH}_{3}\right)_{2}, \mathrm{R}^{\prime \prime}=\mathrm{C}_{6} \mathrm{H}_{5} \mathrm{CH}_{2}, \mathrm{Cl}^{\prime}$.

Scheme. The synthesis of quaternary salts of adamantane-based dialkylaminopropanols (3a-1) 
PHYSICOCHEMICAL PROPERTIES OF COMPOUNDS 3a-31

\begin{tabular}{|c|c|c|c|c|c|c|c|c|c|}
\hline \multirow{2}{*}{ Compound } & \multirow{2}{*}{ M. p., ${ }^{\circ} \mathrm{C}$} & \multirow{2}{*}{ Yield, \% } & \multicolumn{3}{|c|}{ Found, $\%$} & \multirow{2}{*}{ Mol. Formula } & \multicolumn{3}{|c|}{ Calc, \% } \\
\hline & & & $\mathrm{C}$ & $\mathrm{H}$ & $\mathrm{N}$ & & $\mathrm{C}$ & $\mathrm{H}$ & $\mathrm{N}$ \\
\hline $3 \mathbf{a}$ & $97-99$ & 51 & 55.34 & 8.44 & 2.93 & $\mathrm{C}_{22} \mathrm{H}_{40} \mathrm{NO}_{2} \cdot \mathrm{I}^{-}$ & 55.32 & 8.41 & 2.92 \\
\hline $3 c$ & $167-168$ & 64 & 70.64 & 9.38 & 3.43 & $\mathrm{C}_{24} \mathrm{H}_{38} \mathrm{NO}_{2} \cdot \mathrm{Cl}^{-}$ & 70.60 & 9.30 & 3.40 \\
\hline $3 d$ & $128-131$ & 73 & 53.45 & 8.07 & 3.11 & $\mathrm{C}_{20} \mathrm{H}_{36} \mathrm{NO}_{2} \mathrm{I}^{-}$ & 53.40 & 8.00 & 3.07 \\
\hline $3 f$ & $123-125$ & 52 & 72.77 & 9.59 & 3.02 & $\mathrm{C}_{28} \mathrm{H}_{44} \mathrm{NO}_{2} \mathrm{Cl}^{-}$ & 72.77 & 9.60 & 3.00 \\
\hline $3 g$ & $138-141$ & 63 & 51.31 & 7.65 & 3.32 & $\mathrm{C}_{18} \mathrm{H}_{32} \mathrm{NO}_{2} \mathrm{I}^{-}$ & 51.27 & 7.66 & 3.33 \\
\hline $3 \mathrm{~h}$ & 157-159 & 68 & 52.41 & 7.87 & 3.21 & $\mathrm{C}_{19} \mathrm{H}_{34} \mathrm{NO}_{2} \mathrm{I}^{-}$ & 52.41 & 7.84 & 3.20 \\
\hline $3 \mathbf{i}$ & 174-175 & 70 & 50.12 & 7.42 & 3.43 & $\mathrm{C}_{17} \mathrm{H}_{30} \mathrm{NO}_{2} \mathrm{I}^{-}$ & 50.10 & 7.39 & 3.41 \\
\hline $3 \mathbf{j}$ & $172-174$ & 60 & 69.54 & 9.01 & 3.68 & $\mathrm{C}_{22} \mathrm{H}_{34} \mathrm{NO}_{2} \mathrm{Cl}^{-}$ & 69.50 & 9.00 & 3.63 \\
\hline
\end{tabular}

During our synthetic work, 12 new compounds of adamantane-based dialkylaminopropanol quaternary salts were obtained. Compounds are colorless or light yellow substances, soluble in water and in organic solvents (DMSO, ethanol).

The melting point, elemental analysis and yield of compounds (3a-3l) are shown in Table 1 . The data obtained from the calculation of elemental analysis $(\mathrm{C}, \mathrm{N}, \mathrm{H})$ are corresponded to experimental data.

In the IR-spectra of synthesized compounds the adsorption bands due to the $\mathrm{OH}$-group are present in the range of 3500-3200 $\mathrm{cm}^{-1}$, the adsorption bands of $\mathrm{CH}_{2}$ $\mathrm{CH}_{3}$-groups are in $2975-2840 \mathrm{~cm}^{-1}$ region. They also show adsorptions near $1150-1100 \mathrm{~cm}^{-1}$ due to stretching of ether linkage.

All ${ }^{1} \mathrm{H}$ NMR-spectra of compounds contain the signals of protons of adamantane ring at $1.50-2.10 \mathrm{ppm}$; the $\mathrm{CH}_{2}$ group of benzyl radical gives the resonance peaks at 4.75-5.15 ppm as a doublet of doublets. The protons in the benzene ring resonate in the region of 7.40-7.65 ppm, the ethyl group displays a triplet at 3.75-4.11 ppm. The ${ }^{1} \mathrm{H}$ NMR data of compounds are given in Table 2.

Table 2

THE DATA OF ${ }^{1} \mathrm{H}$ NMR-SPECTRAL OF COMPOUNDS 3a-31

\begin{tabular}{|c|c|c|c|c|}
\hline \multirow{2}{*}{ Compound } & \multicolumn{4}{|c|}{ Chemical shift, $\delta$, ppm. $\left({ }^{1} \mathrm{H}\right.$ NMR DMSO-d ${ }_{6}, \delta$, ppm $)$} \\
\hline & Ad & $\mathrm{OH}$ & Ar & Other groups \\
\hline 1 & 2 & 3 & 4 & 5 \\
\hline $3 \mathbf{b}$ & $\begin{array}{l}1.48 \mathrm{~s}\left(6 \mathrm{H}, 3 \mathrm{xCH}_{2}\right) \\
1.62 \mathrm{q}\left(6 \mathrm{H}, 3 \mathrm{xCH}_{2}\right) \\
1.91 \mathrm{~s}(3 \mathrm{H}, 3 \mathrm{xCH})\end{array}$ & $5.54 \mathrm{~d}$ & & $\begin{array}{c}1.31 \mathrm{t}\left(\left(2 \mathrm{H}, \mathrm{CH}_{2} \mathrm{Ad}\right), 3.11 \mathrm{~s}\left(9 \mathrm{H}, \mathrm{N}\left(\mathrm{CH}_{3}\right)_{3}\right), 3.42 \mathrm{~m}\left(2 \mathrm{H}, \mathrm{CH}_{2} \mathrm{O}\right)\right. \\
3.51 \mathrm{~m}\left(2 \mathrm{H}, \mathrm{CH}_{2} \mathrm{~N}\right), 3.90 \mathrm{~m}\left(2 \mathrm{H}, \mathrm{OCH}_{2}\right), 4.43 \mathrm{~m}(1 \mathrm{H}, \mathrm{CH})\end{array}$ \\
\hline $3 c$ & $\begin{array}{l}1.48 \mathrm{~s}\left(6 \mathrm{H}, 3 \mathrm{xCH}_{2}\right) \\
1.62 \mathrm{q}\left(6 \mathrm{H}, 3 \mathrm{xCH}_{2}\right) \\
1.91 \mathrm{~s}(3 \mathrm{H}, 3 \times \mathrm{xCH}) \\
\end{array}$ & $6.07 \mathrm{~d}$ & $\begin{array}{c}7.44 \mathrm{~m}, 7.62 \mathrm{~m} \\
\left(5 \mathrm{H}, \mathrm{C}_{6} \mathrm{H}_{5}\right)\end{array}$ & $\begin{array}{c}1.32 \mathrm{t}\left(2 \mathrm{H}, \mathrm{CH}_{2} \mathrm{Ad}\right), 3.08 \mathrm{~s}, 3.12 \mathrm{~s}\left(6 \mathrm{H}, \mathrm{N}\left(\mathrm{CH}_{3}\right)_{2}\right), 3.40 \mathrm{~m}\left(2 \mathrm{H}, \mathrm{CH}_{2} \mathrm{O}\right), \\
3.50 \mathrm{~m}\left(2 \mathrm{H}, \mathrm{CH}_{2} \mathrm{~N}\right), 3.91 \mathrm{dd}, 3.99 \mathrm{dd}\left(2 \mathrm{H}, \mathrm{OCH}_{2}\right), 4.58 \mathrm{~s}(1 \mathrm{H}, \mathrm{CH}), \\
4.72 \mathrm{q}\left(2 \mathrm{H}, \mathrm{CH}_{2} \mathrm{C}_{6} \mathrm{H}_{5}\right)\end{array}$ \\
\hline $3 d$ & $\begin{array}{l}1.49 \mathrm{~s}\left(6 \mathrm{H}, 3 \mathrm{xCH}_{2}\right) \\
1.63 \mathrm{q}\left(6 \mathrm{H}, 3 \mathrm{xCH}_{2}\right) \\
1.90 \mathrm{~s}(3 \mathrm{H}, 3 \mathrm{xCH})\end{array}$ & $5.48 \mathrm{~d}$ & & $\begin{array}{c}1.32 \mathrm{t}\left(\left(2 \mathrm{H}, \mathrm{CH}_{2} \mathrm{Ad}\right), 2.04 \mathrm{~m}\left(4 \mathrm{H},\left(\mathrm{CH}_{2}\right)_{2} \text { pyrrole }\right), 3.11 \mathrm{~s}\left(3 \mathrm{H}, \mathrm{NCH}_{3}\right),\right. \\
3.42-3.60 \mathrm{~m}\left(6 \mathrm{H}, \mathrm{N}\left(\mathrm{CH}_{2}\right)_{3}\right), 3.41 \mathrm{~m}\left(2 \mathrm{H}, \mathrm{CH}_{2} \mathrm{O}\right), 4.52 \mathrm{~m}(1 \mathrm{H}, \mathrm{CH})\end{array}$ \\
\hline $3 f$ & $\begin{array}{l}1.49 \mathrm{~s}\left(6 \mathrm{H}, 3 \mathrm{xCH}_{2}\right) \\
1.65 \mathrm{q}\left(6 \mathrm{H}, 3 \mathrm{xCH}_{2}\right) \\
1.91 \mathrm{~s}(3 \mathrm{H}, 3 \mathrm{xCH})\end{array}$ & $6.15 \mathrm{~d}$ & $\begin{array}{l}7.43 \mathrm{~m}, 7.64 \mathrm{~m} \\
\left(5 \mathrm{H}, \mathrm{C}_{6} \mathrm{H}_{5}\right)\end{array}$ & $\begin{array}{c}1.31 \mathrm{t}\left(2 \mathrm{H}, \mathrm{CH}_{2} \mathrm{Ad}\right), 1.63-1.92 \mathrm{~m}\left(8 \mathrm{H},\left(\mathrm{CH}_{2}\right)_{4}\right), 3.43-3.80 \mathrm{~m} \\
\left(6 \mathrm{H}, \mathrm{N}\left(\mathrm{CH}_{2}\right)_{3}\right), 3.41 \mathrm{~m}\left(2 \mathrm{H}, \mathrm{CH}_{2} \mathrm{O}\right), 4.41 \mathrm{~m}(1 \mathrm{H}, \mathrm{CH}) \\
4.50 \mathrm{q}\left(2 \mathrm{H}, \mathrm{CH}_{2} \mathrm{C}_{6} \mathrm{H}_{5}\right)\end{array}$ \\
\hline $3 g$ & $\begin{array}{l}1.58 \mathrm{~s}\left(6 \mathrm{H}, 3 \mathrm{xCH}_{2}\right) \\
1.68 \mathrm{q}\left(6 \mathrm{H}, 3 \mathrm{xCH}_{2}\right) \\
2.10 \mathrm{~s}(3 \mathrm{H}, 3 \mathrm{xCH}) \\
\end{array}$ & $5.76 \mathrm{~d}$ & & $\begin{array}{c}2.06 \mathrm{~m}\left(4 \mathrm{H},\left(\mathrm{CH}_{2}\right)_{2}\right), 3.10 \mathrm{~s}\left(3 \mathrm{H}, \mathrm{NCH}_{3}\right), 3.30-3.70 \mathrm{~m}\left(8 \mathrm{H}, \mathrm{N}\left(\mathrm{CH}_{2}\right)_{3} ;\right. \\
\left.\mathrm{OCH}_{2}\right), 3.97 \mathrm{~m}(1 \mathrm{H}, \mathrm{CH})\end{array}$ \\
\hline $3 \mathbf{h}$ & $\begin{array}{l}1.57 \mathrm{~s}\left(6 \mathrm{H}, 3 \mathrm{xCH}_{2}\right) \\
1.67 \mathrm{q}\left(6 \mathrm{H}, 3 \mathrm{xCH}_{2}\right) \\
2.09 \mathrm{~s}(3 \mathrm{H}, 3 \mathrm{xCH})\end{array}$ & $5.54 \mathrm{~d}$ & & $\begin{array}{c}1.25 \mathrm{t}\left(3 \mathrm{H}, \mathrm{CH}_{3}\right), 2.05 \mathrm{~m}\left(4 \mathrm{H},\left(\mathrm{CH}_{2}\right)_{2}\right), 3.21 \mathrm{q}\left(2 \mathrm{H}, \mathrm{CH}_{2} \mathrm{CH}_{3}\right) \\
3.30-3.70 \mathrm{~m}\left(8 \mathrm{H}, \mathrm{N}\left(\mathrm{CH}_{2}\right)_{3} ; \mathrm{OCH}_{2}\right), 3.97 \mathrm{~m}(1 \mathrm{H}, \mathrm{CH})\end{array}$ \\
\hline
\end{tabular}


Continuation of Table 2

\begin{tabular}{|c|c|c|c|c|}
\hline 1 & 2 & 3 & 4 & 5 \\
\hline $3 i$ & $\begin{array}{l}1.58 \mathrm{~s}\left(6 \mathrm{H}, 3 \mathrm{xCH}_{2}\right) \\
1.68 \mathrm{q}\left(6 \mathrm{H}, 3 \mathrm{xCH}_{2}\right) \\
2.10 \mathrm{~s}(3 \mathrm{H}, 3 \mathrm{xCH})\end{array}$ & $6.08 \mathrm{~d}$ & & $\begin{array}{c}3.11 \mathrm{~s}\left(9 \mathrm{H}, \mathrm{N}\left(\mathrm{CH}_{3}\right)_{3}\right), 3.50 \mathrm{~m}\left(2 \mathrm{H}, \mathrm{CH}_{2} \mathrm{~N}\right), 3.90 \mathrm{~m}\left(2 \mathrm{H}, \mathrm{OCH}_{2}\right) \\
4.13 \mathrm{~m}(1 \mathrm{H}, \mathrm{CH})\end{array}$ \\
\hline $3 \mathbf{j}$ & $\begin{array}{l}1.57 \mathrm{~s}\left(6 \mathrm{H}, 3 \mathrm{xCH}_{2}\right) \\
1.67 \mathrm{q}\left(6 \mathrm{H}, 3 \mathrm{xCH}_{2}\right) \\
2.09 \mathrm{~s}(3 \mathrm{H}, 3 \mathrm{xCH})\end{array}$ & $5.73 \mathrm{~d}$ & $\begin{array}{l}7.44 \mathrm{~m}, 7.63 \mathrm{~m} \\
\left(5 \mathrm{H}, \mathrm{C}_{6} \mathrm{H}_{5}\right)\end{array}$ & $\begin{array}{c}3.08 \mathrm{~s}, 3.12 \mathrm{~s}\left(6 \mathrm{H}, \mathrm{N}\left(\mathrm{CH}_{3}\right)_{2}\right), 3.52 \mathrm{~m}\left(2 \mathrm{H}, \mathrm{CH}_{2} \mathrm{~N}\right), 3.98 \mathrm{~m}\left(2 \mathrm{H}, \mathrm{OCH}_{2}\right), \\
4.20 \mathrm{~m}(1 \mathrm{H}, \mathrm{CH}), 4.73 \mathrm{q}\left(2 \mathrm{H}, \underline{\mathrm{CH}}_{2} \mathrm{C}_{6} \mathrm{H}_{5}\right)\end{array}$ \\
\hline $3 k$ & $\begin{array}{l}1.58 \mathrm{~s}\left(6 \mathrm{H}, 3 \mathrm{xCH}_{2}\right) \\
1.68 \mathrm{q}\left(6 \mathrm{H}, 3 \mathrm{xCH}_{2}\right) \\
2.09 \mathrm{~s}(3 \mathrm{H}, 3 \mathrm{xCH})\end{array}$ & $5.57 \mathrm{~d}$ & & $\begin{array}{c}3.11 \mathrm{~s}\left(9 \mathrm{H}, \mathrm{N}\left(\mathrm{CH}_{3}\right)_{3}\right), 3.34 \mathrm{~m}\left(2 \mathrm{H}, \mathrm{CH}_{2} \mathrm{~N}\right), 3.54 \mathrm{~m}\left(2 \mathrm{H}, \mathrm{OCH}_{2}\right), 3.74 \mathrm{t} \text {, } \\
4.08 \mathrm{t}\left(4 \mathrm{H},\left(\mathrm{CH}_{2}\right)_{2}\right), 4.29 \mathrm{~m}(1 \mathrm{H}, \mathrm{CH})\end{array}$ \\
\hline 31 & $\begin{array}{l}1.58 \mathrm{~s}\left(6 \mathrm{H}, 3 \mathrm{xCH}_{2}\right) \\
1.68 \mathrm{q}\left(6 \mathrm{H}, 3 \mathrm{xCH}_{2}\right) \\
2.09 \mathrm{~s}(3 \mathrm{H}, 3 \mathrm{xCH})\end{array}$ & $5.78 \mathrm{~d}$ & $\begin{array}{l}7.43 \mathrm{~m}, 7.64 \mathrm{~m} \\
\left(5 \mathrm{H}, \mathrm{C}_{6} \mathrm{H}_{5}\right)\end{array}$ & $\begin{array}{c}3.08 \mathrm{~s}, 3.12 \mathrm{~s}\left(6 \mathrm{H}, \mathrm{N}\left(\mathrm{CH}_{3}\right)_{2}\right), 3.50 \mathrm{~m}\left(2 \mathrm{H}, \mathrm{OCH}_{2}\right), 3.76 \mathrm{t}, 4.12 \mathrm{t} \\
\left(4 \mathrm{H},\left(\mathrm{CH}_{2}\right)_{2}\right), 4.22 \mathrm{~m}(1 \mathrm{H}, \mathrm{CH}), 4.75 \mathrm{q}\left(2 \mathrm{H}, \underline{\mathrm{CH}}_{2} \mathrm{C}_{6} \mathrm{H}_{5}\right)\end{array}$ \\
\hline
\end{tabular}

1-(1-adamantylethoxy)-3-(N-methyl hexamethylenamine)-2-propanol iodide (3a). To the mixture of 1-adamantylethyl glycidyl ether $(2.36 \mathrm{~g} / 0.01 \mathrm{Mol})$ in isopropanol (5 ml), hexamethylenamine $(1.48 \mathrm{~g} / 0.015 \mathrm{Mol})$ was added, and the reaction mixture was heated for $8 \mathrm{~h}$. The excess of amine and alcohol was evaporated under reduced pressure. The residue was dissolved in $5 \mathrm{ml}$ of acetonitrile with adding methyl iodide $(0.75 \mathrm{~g} / 0.0105 \mathrm{Mol})$ followed refluxing for $10 \mathrm{~h}$. After cooling to the appropriate temperature, dry diethyl ether $(5 \mathrm{ml})$ was added, than the reactive mixture was left for $6-8 \mathrm{~h}$ at $+5^{\circ} \mathrm{C}$. The precipitate was filtered out, washed with diethyl ether and dried. Yield $-2.43 \mathrm{~g}(51 \%)$. M. p. $-97-99^{\circ} \mathrm{C}$.

For compounds $\mathbf{3 b}$-31 all procedures were the same.

\section{Experimental Biological Part}

Antibacterial and antifungal activity assay showed that derivatives, containing adamatylethhyl radical in their alkoxy group (3a-3f), possessed significant inhibitory activity (Table 3).

No antimicrobial activity was observed against tested bacterial and fungal strains for compounds, containing 1-adamantyl (3g-3j) and 1-adamantyloxyethyl (3k-3i) fragment in their alkoxy group (except compound 31 with activity against $S$. aureus, MIC $50.0 \mu \mathrm{g} / \mathrm{mL}$ ).

Among the adamantane-based derivatives tested, compounds 3a-f and $\mathbf{3} \mathbf{l}$ possessed inhibitory activity against the grampositive bacteria (S. aureus), the MIC values were between 1.25 and $50.0 \mu \mathrm{g} / \mathrm{mL}$. The most active compounds were $\mathbf{3 c}$ and $\mathbf{3 e}$, which inhibited $S$. aureus as well as myramistin.

Derivatives of adamantane were also active when tested against gramnegative strains. The compounds 3a-c,e,f at concentrations between 3.12 and $50.0 \mu \mathrm{g} / \mathrm{mL}$ inhibited $E$. coli growth. The MIC value of compound $\mathbf{3 c}$ was comparable to that of myramistin.

Antifungal activity results revealed that compounds 3a-f possessed inhibitory action against $C$. albicans at concentrations less than or equal to $25.0 \mu \mathrm{g} / \mathrm{mL}$. The compounds $\mathbf{3 c}$ and $\mathbf{3 f}$, as well as myramistin, inhibited the yeast growth (MICs 1.56 and $1.25 \mu \mathrm{g} / \mathrm{mL}$ respectively).

In conclusion, this study showed that $\mathbf{3 c}$ was the most active compound, and its antimicrobial effect was simi-

Table 3

ANTIMICROBIAL ACTIVITY OF COMPOUNDS 3a-3l

\begin{tabular}{|c|c|c|c|}
\hline \multirow[b]{2}{*}{ Compound } & \multicolumn{3}{|c|}{ MIC, $\mu \mathrm{g} / \mathrm{mL}$} \\
\hline & $\begin{array}{c}\text { S. aureus } \\
\text { ATCC } 25923\end{array}$ & $\begin{array}{c}\text { E. coli } \\
\text { ATCC } 25922\end{array}$ & $\begin{array}{c}\text { C. albicans } \\
\text { NCTC } 885 / 653\end{array}$ \\
\hline $3 a$ & 25.0 & 50.0 & 25.0 \\
\hline $3 \mathbf{b}$ & 12.5 & 50.0 & 25.0 \\
\hline $3 \mathbf{c}$ & 2.5 & 3.12 & 1.56 \\
\hline $3 d$ & 20.0 & $>50.0$ & 10.0 \\
\hline $3 \mathbf{e}$ & 1.25 & 25.0 & 12.5 \\
\hline $3 f$ & 12.5 & 12.5 & 1.56 \\
\hline $3 g$ & $>50.0$ & $>50.0$ & $>50.0$ \\
\hline $3 \mathrm{~h}$ & $>50.0$ & $>50.0$ & $>50.0$ \\
\hline $3 \mathbf{i}$ & $>50.0$ & $>50.0$ & 50.0 \\
\hline $3 \mathbf{j}$ & $>50.0$ & $>50.0$ & $>50.0$ \\
\hline $3 \mathrm{k}$ & $>50.0$ & $>50.0$ & $>50.0$ \\
\hline 31 & 50.0 & $>50.0$ & $>50.0$ \\
\hline Myramistin & 2.5 & 5.0 & 1.25 \\
\hline
\end{tabular}


lar to that of myramistin. Significant inhibitory activity against $S$. aureus and C. albicans was registered for compounds $\mathbf{3 e}$ and $\mathbf{3 f}$ respectively. So, quaternary salts of dialkylaminopropanol with 1-adamantylethyl radical in their alkoxy group possessed polyvalent action against bacteria and fungi. These compounds are promising class for the research and development of novel antimicrobial agents for treatment of infectious diseases.

\section{CONCLUSIONS}

1. The effective synthetic methods of 12 novel quaternary salts of adamantane-containing dialkylaminopropanol and their derivatives have been developed.

2. The structure of the compounds obtained has been confirmed with a set of modern physical and chemical me- thods of analysis, and their individuality has been proven by elemental analisys, IR- and NMR-spectroscopy.

3. It was shown that compounds with 1-adamatylethhyl radical in their alkoxy group (3a-3f) possessed narrow (3e and 3f) and broad spectrum (3c) of antimicrobial action against bacteria and fungi.

4. It was find that derivatives with 1-adamantyl (3g-3j) and 1-adamantyloxyethyl (3k-3l) radical in their alkoxy group had no antimicrobial activity against bacterial and fungal strains.

5. The present results suggest that compound $3 \mathbf{c}$ could be a lead for development of antimicrobial agents in the future.

Conflict of Interests: authors have no conflict of interests to declare.

\section{REFERENCES}

1. WHO's first global report on antibiotic resistance reveals serious, worldwide threat to public health. - [Електронний ресурс] - Режим доступу: http://www.who.int/mediacentre/news/releases/2014/amr-report/en/.

2. Синтез, антимикробная активность 1-(4-(1-адамантил)фенокси)-3-амино-2-пропанола / Ю. В. Короткий, Н. А. Врынчану, Ю. Н. Максимов и др. // Хим.-фарм. журн. - 2009. - Т. 43, № 6. - С. 10-13. https://doi.org/10.1007/s11094-009-0299-7

3. Синтез, антимикробная и противогрибковая активность четвертичных солей адамантансодержащих алкоксидиалкиламинопропанолов / Ю. В. Короткий, Н. А. Врынчану, Ю. Н. Максимов и др. // Хим.-фарм. журн. - 2011. - Т. 45, № 1. - С. 21-23. https://doi.org/10.1007/ s11094-011-0552-8

4. Морозов, И. С. Фармакология адамантанов / И. С. Морозов, В. И. Петров, С. А. Сергеева. - Волгоград: Волгоград. мед. акад., - 2001. - 320.с

5. Определение чувствительности микроорганизмов к антибактериальным препаратам: методические указания МУК 4.2.1890-04 / Н. А. Семина, С. В. Сидоренко, С. П. Резван и др. // Клин. микробиол. и антимикроб. химиотерап. - 2004. - Т. 6, № 4. - С. $306-359$.

6. Синтез и противовирусная активность адамантилоксиранов и их производных / А. К. Ширяев, И. К. Моисеев, Е. И. Боряко и др. // Хим.фарм. журн. - 1990. - Т. 24, № 5. - С. 23-25.

7. A convenient one-step synthesis of glycidyl ethers / G. Mouzin, H. Cousse, J. P. Rieu et al. // Synthesis. - 1983. - № 2 (1983). - P. 117-119. https:// doi.org/10.1055/s-1983-30243

\section{REFERENCES}

1. WHO's first global report on antibiotic resistance reveals serious, worldwide threat to public health. (2014). World Health Organization. who.int Available from: http://www.who.int/mediacentre/news/releases/2014/amr-report/en/

2. Korotkii, Y. V., Vrynchanu, N. A., Maksimov, Y. N., Lozinskii, M. O. (2009). Synthesis, antimicrobial activity of 1-(4-(1-adamantyl)-phenoxy)-3-amino2-propanol. Khimiko-farmatsevtichesky zhurnal - Pharmaceutical Chemistry Journal, 43 (6), 10-13. https://doi.org/10.1007/s11094-009-0299-7

3. Korotkii, Y. V., Vrynchanu, N. A., Maksimov, Y. N., Lozinskii, M. O. (2011). Synthesis, antimicrobial and antifungal activities of quaternary salts of adamantane-containing alkoxydialkyl aminopropanols. Khimiko-farmatsevtichesky zhurnal - Pharmaceutical Chemistry Journal, 45 (1), $21-23$. https://doi.org/10.1007/s11094-011-0552-8

4. Morozov, I. S., Petrov, V. I., Sergeeva, S. A. (2001). Pharmacology of adamantanes. Volgograd: Volgogradskaya med. academiya, 320.

5. Semina, N. A., Sidorenko, S. V., Rezvan, S. P. Grudinina S. A., ... Sreda, Z. S. (2004). Opredelenie chuvstvitel'nosti mikroorganizmov k antibakterial'nym preparatam. Metodicheskie Ukazaniya MUK, 4, 1890-04.

6. Shiryaev, A. K., Moiseev, I. K., Boryako, E. I., Korobchenko, L. V., Vladyko, G. V. (1990). Synthesis and antiviral activity of adamantyloxiranes and their derivatives. Khimiko-farmatsevtichesky zhurnal - Pharmaceutical Chemistry Journal, 24 (5), 23-25.

7. Mouzin, G., Cousse, H., Rieu, J. P., Duflos, A. (1983). A convenient one-step synthesis of glycidyl ethers. Synthesis, 1983 (02), 117-119. https:// doi.org/10.1055/s-1983-30243

\section{Відомості про авторів:}

Короткий Ю. В., канд. фарм. наук, зав. відділу трансферу технологій, інноваційної діяльності та інтелектуальної власності, Інститут органічної хімії НАН України. E-mail: ioch@ioch.kiev.ua. ORCID: http://orcid.org/0000-0003-4475-1298

Дудікова Д. М., молодший наук. співробітник лабораторії фармакології протимікробних засобів, ДУ «Інститут фармакології та токсикології HAMН України». E-mail: darmardud@gmail.com. ORCID: http://orcid.org/0000-0002-4733-1267

Вринчану Н. О., д-р. мед. наук, зав. лабораторії фармакології протимікробних засобів, ДУ «Інститут фармакології та токсикології

HAMН України». E-mail: nvrynchanu@gmail.com. ORCID: http://orcid.org/0000-0003-3450-2108

Смертенко О. А., провідний інженер відділу фізико-хімічних досліджень, Інститут органічної хімії НАН України.

E-mail: olena.smertenko@gmail.com. ORCID: http://orcid.org/0000-0002-9241-3966

Information about authors:

Korotkyi Yu. V., PhD in Pharmacy, Head of the Department of Technology Transfer, Innovation and Intellectual Property,

Institute of Organic Chemistry of the NAS of Ukraine. E-mail: ioch@ioch.kiev.ua. ORCID: http://orcid.org/0000-0003-4475-1298

Dudikova D. M., Junior Researcher of the Laboratory of Pharmacology of Antimicrobial Agents, SI "Institute of Pharmacology and Toxicology

of NAMS of Ukraine". E-mail: darmardud@gmail.com. ORCID: http://orcid.org/0000-0002-4733-1267

Vrynchanu N. O., Doctor of Medicine, Head of the Laboratory of Pharmacology of Antimicrobial Agents, SI "Institute of Pharmacology

and Toxicology of NAMS of Ukraine". E-mail: nvrynchanu@gmail.com. ORCID: http://orcid.org/0000-0003-3450-2108

Smertenko 0. A., Senior Engineer of the Department of Physicochemical Investigations, Institute of Organic Chemistry of the NAS of Ukraine.

E-mail: olena.smertenko@gmail.com. ORCID: http://orcid.org/0000-0002-9241-3966

Сведения об авторах:

Короткий Ю. В., канд. фарм. наук, зав. отделом трансфера технологий, инновационной деятельности и интеллектуальной собственности,

Институт органической химии НАН Украины. E-mail: ioch@ioch.kiev.ua. ORCID: http://orcid.org/0000-0003-4475-1298

Дудикова Д. М., младший научный сотрудник лаборатории фармакологии противомикробных средств, ГУ «Институт фармакологии

и токсикологии HAMН Украины». E-mail: darmardud@gmail.com. ORCID: http://orcid.org/0000-0002-4733-1267

Врынчану Н. А., д-р мед. наук, зав. лабораторией фармакологии противомикробных средств, ГУ «Институт фармакологии

и токсикологии НАMН Украины». E-mail: nvrynchanu@gmail.com. ORCID: http://orcid.org/0000-0003-3450-2108

Смертенко Е. А., ведущий инженер отдела физико-химических исследований, Институт органической химии НАН Украины.

E-mail: olena.smertenko@gmail.com. ORCID: http://orcid.org/0000-0002-9241-3966 